\title{
Editorial
}

\section{Authorship in Scientific Medical Journals}

Birdem Med J 2016; 6(1): 1-6

Scientific paper authorship is an important academic achievement for all research professionals. To become designated as an author of a paper has academic, research, social and financial implications. While authors get credit for the published work, they must accept the public responsibility that goes with it.In an endeavor to ensure honest practice, the International Committee of Medical Journal Editors (ICMJE), the Vancouver group developed the criteria for authorship and said that all persons designated as authors should qualify for authorship and all those who qualify should be listed.

\section{Background}

In academic field a publication may influence job appointments, promotions, posting, fellowship and research project awards. Professionals with strong publication records are considered to be more competent than their lesser published colleagues. This explains why many people go for the gift authorship in manuscripts to which they have not contributed intellectually. The prevalent culture of "publish or perish" has been responsible for the abuse of authorship. The scientific enterprise expanded dramatically after World War II, ushering in what was termed as the "era of big science". The expansion was due in part to the increase of government funding for research, increase in science education, technological development and commercialization of scientific discoveries. As the scientific workforce expanded, so did the number of science publications and number of coauthors on those publications. Authorship is a constant topic for discussion in scientific publishing; attitude to and practices of scientific authorship also varies. Problems about the threshold of involvement quantifying for authorship as well as the order of listing authors are unresolved.

\section{Introduction}

The number of authors per manuscript in peer-reviewed journals has increased substantially in last several decades. It is unknown however whether authorship has grown over time due to growing complexity of published academic articles, in which case growth could be warranted or whether it has grown due to pressure of funding and academic promotion, which have created authorship inflation. Inappropriate authorship occurs frequently. It has been observed that on an average one-third of researchers had experienced misuse of authorship and prevalence of so called honorary authorship was found in one-fourth of original research papers.

\section{Criteria for Authorship}

Criteria have been developed by the International Committee of Medical Journal Editors (ICMJE) to establish standardized definitions and thereby secure responsibility and accountability of authorship in medical journals. Hundreds of journals have adopted these criteria, but controversies still exist and breaches of authorship guidelines are common. Fact is, it has been said on responsible conduct of research, that "no issue is more ubiquitous and contentious than the question of authorship".

Authorship should be based on the following 4 criteria:

1. Substantial contribution to the conception or design of the work; or the acquisition, analysis or interpretation of data of the work; and

2. Drafting the work or revising it critically for important intellectual content; and

3. Final approval of the version to be published; and

4. Agreement to be accountable to all aspects of the work in ensuring that questions related to the accuracy or integrity of any part of the work are appropriately investigated or resolved.

In addition to being accountable for the parts of the work he or she has done, an author should be able to identify which co-authors are responsible to which specific other parts of the work. In addition, authors should have confidence in the integrity of the contributions of their co-authors. 
"All those designated as authors should meet all four criteria for authorship, and all who meet the four criteria should be identified as authors". The criteria are not intended for use as a means to disqualify colleagues from authorship who otherwise meet authorship criteria by denying them the opportunity to meet criteria 2 or 3 . Therefore, all individuals who meet the first criterion should have the opportunity to participate in the review, drafting and final approval of the manuscript. Individuals who conduct the work are responsible for identifying who meets these criteria and ideally should do so when planning the work.

Examples of contribution that do not qualify for authorship but that should be acknowledged in the paper:

1. Providing funding, technical advice, reagents, samples or patient data

2. Providing students or technical personnel who perform studies

3. Routine collection of data

4. General supervision of the research group

Authorship obviously conveys professional benefit. Students in many biomedical research graduate programme can not earn $\mathrm{PhD}$ without publishing one or more first authored papers; for these and many other reasons there may be abuse of authorship.

\section{Authorship abuse/promiscus authorship}

It means awarding authorship to someone who has not contributed in an intellectually significant way to the paper. It can be of many forms-

\section{Coercive authorship}

It has been defined as authorship conferred to individuals in response to their exertion of seniority or supervisory status over subordinates and junior investigators. This sort of attempt by senior individuals to force their way onto the publications of subordinates and junior investigators in the absence of appropriate intellectual contribution as the "White Bull Effect" and was argued that such behaviour is a form of fraud and scientific misconduct. Authorship coercion may be done by many other intimidation tactics.

\section{Honorary/guest/gift authorship}

It is defined as the awarding of authorship out of respect or friendship, in an attempt to curry favor and/or to give the paper a greater sense of legitimacy.

\section{Mutual support authorship}

It is defined as agreement by two or more investigators to place their names on each other's paper to give the appearance of higher productivity.

\section{Duplication authorship}

It is the publication of the same work in multiple journals. Reward system that emphasize numbers of papers over quality foster "mutual support" and "duplication authorship" abuse.

\section{Ghost authors}

Are authors whose names are omitted from a paper. There can be numerous deceitful reasons for ghost authorship. For example, it is well known that some pharmaceutical companies hire professional writers to write papers favorably describing their products. A bonafide academic is then asked or hired to sign his/her name on the paper to give it and the product legitimacy.

\section{Denial of authorship}

The most typical example of this involves the individuals who participate in generating data for what they presume is a legitimate scientific collaboration. However, the other so called collaborator, publish paper using these data without giving the investigators coauthorship or accurately acknowledging their contribution. Denial of authorship can rightfully be considered a form of plagiarism and therefore a scientific misconduct.

\section{Consequences of Authorship Abuse}

The infamous example of the consequence of promiscus authorship is the Darsee affair. Dr. John Darsee was a clinician investigator who worked at Harvard Medical School and Emory University School of Medicine from 1978 to 1981. The investigator committee at Harvard, Emory and National Institute of Health (NIH) ultimately concluded that more than 100 of his publications contained fabricated data. Another case of fraud and promiscus authorship is that of Robert Slutsky, a clinical investigator at the University of California at San Diego (USCD) from 1983 to 1984, where he published one paper every 10 days. The USCD investigators stated his work a "mockery of authorship of scientific manuscripts". Korean stem cell scandal is another example of promiscuity where Dr. Woo Suk Hwang published two high profile papers in "Science". Dr. Gerald Schatten was a co-author for this. When the work was revealed 
to be fraudulent, Schatten made significant effort to distance himself from it. No evidence was found that Schatten had falsified any aspect of scientific publication, but he was found guilty of scientific misbehaviour. In Academic Medicine, a recent paper defines "scientific misbehavior" as 'problematic' or 'questionable' behaviour and is distinct from scientific misconduct, which is fabrication and falsification of data and plagiarism.

\section{How to minimize and prevent authorship abuse?}

All persons designated as authors should qualify for authorship as per ICMJE authors' requirement guidelines and all those who qualify should be listed. A concern is over the uniform requirement and the definition of "substantial contributions" which has been raised in authorship guidelines. It seems two requirements must be fulfilled - firstly, it is required that all authors participate in the drafting or revising of the manuscript for "important intellectual content". Providing funding, reagents or advice deserves acknowledgement, not authorship. Secondly, it is required that all authors must be able to take public responsibility for the contribution they have made.

Numerous organizations, universities and research institutes also have established formal authorship guidelines. There is a broad consensus in all those guidelines in that authors in scientific papers must have contributed an "intellectually significant" way to the work, they must be able to take the public responsibility for that contribution and they must have participated in writing the manuscript.

\section{Should technicians be authors?}

So long as they have contributed to the paper in an "intellectually significant" way, no harm to be an author. "Rush Medical College Authorship Guideline" state that a technician may be a co-author of a publication, if, in the opinion of the principal investigator, the said individual has made a substantial contribution to the work over and above actually performing required tasks. If the technical studies prescribed by the principal investigator, support staff has performed but has not made contribution to the experimental design, data analysis, data interpretation; rationale for their coauthorship is not automatically earned.

\section{Authorship order and its meaning}

Manuscript authorship and author placement have important implication for accountability and allocation of credit. In most evaluation studies it was found that level of participations were highest for the first authors, followed by the last and then second authors. Middle authors had lower levels of contributions particularly in conception, draft of the manuscript, supervision and being a guarantor.

\section{Dual/co-first authorship}

There has been a dramatic increase in number and percentage of publications in biomedical journal and clinical journal in which two or more co-authors claim first authorship with change in some journals from no joint authorship in 1990 to co-first authorship of $>30 \%$ of all research publications in 2012.To ensure appropriate recognition for equal work, it has been suggested to journals and citation database to begin identifying joint first authors in a way that makes it easy for readers to locate this information. In addition, probably time has come to established a convention wherein authors identify joint first authors of papers they cite in the reference section it will reinforce the equal contribution made by researchers.

Due to lack of standardized reporting of declaration of joint co-authorship, it can be difficult or impossible for a reader of a manuscript(or its abstract in citation database, including PubMed) to determine whether the original investigation is being published by joint first author. Often recognition of additional first authors become evident only if one refers back to the original paper and searches for the information, which can be indicated by asterisks or superscripts in the author affiliation, written in footnote in the first page or documented in a contribution statement at the end of the paper to name the most common methods of reporting. Sample statement include"[author A] and [author B ]contributed equally to this work and should be regarded as joint first authors,",these authors contributed equally to this work/study",or a superscripted character after the author's name with accompanying explanation,e..,"'Denotes co-first authors. Journal may highlight joint first authors of papers by bolding the names of joint first in the cited list of reference appending an annotation that states"author names in bold designated shared co-first authorship. It is for information that the HTML version of paper on a 
website may not contain joint authorship information that is clearly stated in Pub Med.

So both publisher platform like PubMEd,PubMed central, Google Scholar, Scopus,or web of science and platform that provide full text of article in HTML can indicate joint first authorship in reference list with a distinct font,color or italics or bold letters, an asterisks, a supercript or contribution statement or declaration of joint first authorship.

\section{First author}

In most biomedical research paper it is widely accepted that first author is the person who has carried out a majority of the experimental work described in the paper. For example, PhD students are obviously required to perform the bulk of their thesis work and they must contribute significantly for the intellectual development of their project. It follows logically therefore that the student should be first author on at least one or more publications arising from that work. Indeed, many $\mathrm{PhD}$ programme requires publication of at least one first authored paper for graduation.

\section{Senior author}

Senior author/last author is the position on a paper, given to chairman or other senior person, as a gesture of respect and did not have meaning nor is justified; is typically the last person on an authorship list. Senior authorship must not be awarded to someone simply because of their seniority, rank or standing in the field. It must be the individual who generally direct, oversee and guarantee the authenticity of the work reported and implicitly take responsibility of the work's scientific accuracy. Important role of the senior is to take the responsibility for the project as a whole.

\section{Corresponding author}

Corresponding author is an individual charged with communicating with editors and readers, takes the primary responsibility for communication with journal during the manuscript submission, peer review, and publication process and typically ensures that all the journals administrative requirements such as providing details of authorship, order of authorship, ethics committee approval, clinical trial registration documents and gathering conflict of interest forms and statements are properly completed. Corresponding author is typically the first or last author. Although it may be delegated to one or more co-authors.

\section{Middle or contributing authors}

They are those individuals charged listed between first and senior authors.

\section{Requirements and responsibilities of coauthors}

\begin{tabular}{ll}
\hline Author category & Contribution and responsibility to work and publication \\
\hline First author & Fulfills ICMJE authorship criteria. Perform the bulk of the experimental work. \\
Senior author & Fulfills ICMJE authorship criteria. Typically the last person on the authorship \\
& list. Directs, oversees and guarantees the authenticity of the work. Takes \\
& responsibility for the scientific accuracy, valid methodology, analysis and \\
& conclusion of all work described in the paper. Able to explain all of the results \\
& described in the paper. \\
& Fulfills ICMJE authorship criteria. Typically assumed by the first or senior author. \\
Corresponding author & Communicates with editors and readers. Provides specific information on the \\
& contribution of all coauthors to the paper. Ensures that all authors are aware of and \\
& approve the submission of the manuscript, it's content, authorship and order of \\
authorship. & Fulfills ICMJE authorship criteria. Contributors do not rise to those of first or \\
senior author. Order of middle/contributing authors should reflect their relative \\
contribution to the paper.
\end{tabular}

ICMJE: International Committee of Medical journal editors 
Contributorship/gurantorship: Publication of authorship contributions:

Contributorship and guarantorship are concepts that were applied to original research papers. Each contributorship statement should make clear who has contributed what to the planning, conduct and reporting of the work as described in the article and should identify one or occasionally more contributor(s) as being responsible for the overall content as guarantor.

\section{For artcles in BMJ}

Non original research article such as editorials, clinical reviews and education and debate- it is to state that who had the idea for the article, who performed the literature search, who wrote the article and who is guarantor.

For non-research article which includes case reports such as lesson of week, drug points and interactive case report; it is to state that who identified and/or managed the case(s). Publication of coauthor contributions, either in the printed text or as an online supplement to the paper, should be an uniform policy for all journals. Holding the publication of coauthor contributions to high level of accountability would undoubtedly reduce authorship abuse. BMJ lists contributors in two ways. Firstly, it publishes a list of authors' names at the beginning of the paper and secondly, it lists contributors (some of whom may not be included as authors) at the end of the paper, giving details of who did what in planning, conducting and reporting the work. One or more of these contributors are listed as guarantors of the paper.

Researchers must determine among themselves the precise nature of each person's contribution and $B M J$ encourages open discussion among all person and when encounter disagreement among authors it follows guidence from the Committee on Publication Ethics (COPE).

Others: Some journal such as Proceedings of the Natural Academies of Science requires the authors to publish their contribution to the work as footnote in paper. Nature Journals encourage the authors to do so.

\section{Alteration to authorship or contributorship}

Any change in authors and/or contributors after submission must be approved by all authors. This applies to addition, deletion and changes of authors or contributions being attributed differently. Any alteration must be explained to the editor. The editor may contact any of the authors and /or contributor to ascertain whether they have agreed to any alteration.

\section{Group authorship}

It can be categorized as follows - fewer than 2 authors (Editorials, Commentaries, Letters), fewer than 5 authors (Case Reports and Technical Notes), 5-10 authors (retrospective full-length articles), 10-15 authors (prospective, often grant-funded articles), more than 15 authors (reports of task forces, white papers, etc). Among so many authors, it is not uncommon to find individuals whose contributions are minimal and many times questionable.

If there is very large number of authors, it may be sought that whether everyone listed met the ICMJE criteria for authorship. If they did it may be suggested that the authors form a group whose name will appear in the article byline. It is not unusual that authors may be concerned that their work will not be properly recognized if they form a group which is unfound. When a group name for a specific consortium, committee, study group or the like appears in an article byline, the personal names of that group may be published in the article text. Such names are entered as collaborator names for the Medline citation. The National Library of Medicine no longer limits the number of authors listed on MEDLINE (it did before MEDLINE), as long as all meet the ICMJE criteria.

\section{Searching for Authors, Group Authors and Collaborators in PubMed}

The byline is a short phrase or paragraph that indicates the name of the author of an article in books, magazines, newspapers, newsletters, content Web sites, blogs or other publications.

\section{Byline}

The byline commonly appears between the headline and start of the article, prefaced by the word "By" or "From" or other wording. The byline could also appear at the end of the article.

MEDLINE ${ }^{\circledR}$ contains journal citations and abstracts for biomedical literature from around the world. PubMed ${ }^{\circledR}$ provides free access to MEDLINE and links to full text articles when possible.

To search for an author name, use the search tag [au]. For example, jaeger $\mathrm{j}[\mathrm{au}]$. 
To search for a group (corporate) name, use the search tag [cn]. For example, corgi consortium [cn].

To search for a collaborator ("investigator") name, use the search tag [ir]. For example, maher e [ir].

\section{For $B M J$ authors}

a) If authors form a group for the articles, main byline they will also be listed individually as collaborators in the article Medline/Pubmed record; as authors in a group authorship statement at the end of the article on the bmj.com; as contributors in the contributorship statement at the end of the article on the bmj.com

b) However for the $B M J$ research articles with many authors, where those authors do not opt to form a group, $B M J$ will not publish it in print issue of the $B M J$. Such research article will be for online publication only (thebmj.com).

Let us see a research article with group authorship as it appeared in MEDLINE, with all collaborators clearly listed as individual- http://www.ncbl.nlm.nih.gov/ pubmed/20123835 and (1) here is how the individual authors for article and was downloadable to citation manager:

Effect of a collector bag for measurement of postpartum blood loss after vaginal delivery: cluster randomized trial in13 Eurpean country. Wei-Hong Zhang, Catherine Deneux-Tharaux, Peter Brocklehurst, Edmund Jusczak on behalf of the EUPHRATES Group. BMJ 2010; 340::c293,dio:10.1136/bmj.(published 1 February 2010)

(2) What appeared at the end of the article in an authorship statement:

The following are members of the EUPHRATES (European Project on obstratic Hemorrhage, Reduction, Attitude, Trial and Early warning System): Sophie Alexander(project leader,Belgium),Diogo Ayres-deCampos(Portgal) and so on....

\section{Duplicate/salami authorship}

When the same content is republished in successive papers.It is called duplicate. An acceptable form of duplicate publication may be of addressing a variety of audiences; for example, a paper being published in a second journal because the one in which it was originally published is not available in the country. But in all acses duplicate publication requires written permission from initial journal editorial office because of copyright issue. Howevere both practices divided and duplicate publication are unethical and considered as serious misconduct. COPE has clear guideline for this.

\section{Acknowledgement}

Anyone without qualifying for authorship-has made a notable personal contribution to a publication can be mentioned in acknowledgement;same applies to anybody who made a publication through other significant contribution. A medical writer should always be mentioned in acknowledgement and it should specify the type of contribution.

\section{Conclusion}

Being familiar with authorship requirement,even knowing the breach of it unethical, high proportion of experienced are breaches. It may be due to liberal approach by experienced researcher to authorship. The ICMJE principles should be strictly followed for junior and senior researchers. Medical education, workshop on research protocol may help improve the present trend especially among juniors.

\section{References}

1. Strange K. Authorship: why not just toss a coin? Am J Physiol Cell Physiol 2008;295(3): C567-C575.

2. Baerlocher MO, Newton M, Gautam T, Tomlinson G, Detsky AS. The meaning of author order in medical research. J Investig Med 2007;55(4):174-80.

3. Authorship \& contributorship.available from: www.bmj. com/about-bmj/resources-authors/.../authorshipcontributorshi... (accessed November 30, 2015).

4. Conte ML, Maat SL, Omary MB. Increased co-first authorships in biomedical and clinical publications: a call for recognition. The FASEB Journal article fj.13-235630. Published online July 9, 2013. (accessed November 30, 2015). 